\title{
Short-term developmental outcomes in neonates born to mothers with COVID-19 from Wuhan, China
}

\author{
Ling-Kong Zeng ${ }^{1} \cdot$ Hua-Ping Zhu ${ }^{2} \cdot$ Tian-Tian Xiao ${ }^{3,4} \cdot$ Si-Cong Peng ${ }^{2} \cdot$ Wen-Hao Yuan ${ }^{1}$. Jian-Bo Shao ${ }^{1}$. \\ Lai-Shuan Wang ${ }^{5}$. Fei-Fan Xiao ${ }^{3} \cdot$ Shi-Wen $\mathrm{Xia}^{2} \cdot$ Shoo K. Lee ${ }^{6,7} \cdot \mathrm{Kai} \mathrm{Yan}^{3} \cdot$ Wen-Hao Zhou ${ }^{3}$
}

Received: 4 October 2020 / Accepted: 11 March 2021 / Published online: 12 April 2021

(c) Children's Hospital, Zhejiang University School of Medicine 2021

\begin{abstract}
Background Coronavirus disease 2019 (COVID-19) caused by severe acute respiratory syndrome coronavirus 2 (SARS$\mathrm{CoV}-2$ ) is an emerging disease. The consequences of SARS-CoV-2 exposure in infants remain unknown. Therefore, this study aims to investigate whether neonates born to mothers with COVID-19 have adverse brain development.

Methods This multicenter observational study was conducted at two designated maternal and children's hospitals in Hubei Province, mainland China from February 1, 2020 to May 15, 2020. Neonates born to mothers with COVID-19 were enrolled. Brain magnetic resonance imaging (MRI) findings, and volumes of grey and white matters, and physical growth parameters were observed at 44 weeks corrected gestational age.

Results Of 72 neonates born to mothers with COVID-19, 8 (11\%) were diagnosed with COVID-19, 8 (11\%) were critically ill, and no deaths were reported. Among the eight neonates that underwent brain MRI at corrected gestational age of 44 weeks, five neonates were diagnosed with COVID-19. Among these five neonates, three presented abnormal MRI findings including abnormal signal in white matter and delayed myelination in newborn 2, delayed myelination and brain dysplasia in newborn 3, and abnormal signal in the bilateral periventricular in newborn 5. The other three neonates without COVID-19 presented no significantly changes of brain MRI findings and the volumes of grey matter and white matter compared to those of healthy newborns at the equivalent age $(P>0.05)$. Physical growth parameters for weight, length, and head circumference at gestational age of 44 weeks were all above the 3rd percentile for all neonates.

Conclusions Some of the neonates born to mothers with COVID-19 had abnormal brain MRI findings but these neonates did not appear to have poor physical growth. These findings may provide the information on the follow-up schedule on the neonates exposed to SARS-CoV-2, but further study is required to evaluate the association between the abnormal MRI findings and the exposure to SARS-CoV-2.
\end{abstract}

Keywords Coronavirus disease $2019 \cdot$ Follow-up $\cdot$ Neonate $\cdot$ Severe acute respiratory syndrome coronavirus 2

Shoo K. Lee

shoo.lee@sinaihealthsystem.ca

1 Department of Neonatology, Wuhan Children's Hospital (Wuhan Maternal and Child Healthcare Hospital),

Tongji Medical College, Huazhong University

of Science and Technology,

Wuhan, China

2 Department of Neonatology, Maternal and Child Health Hospital of Hubei Province, Wuhan, China

3 National Children's Medical Center, Children's Hospital of Fudan University, Shanghai, China
4 Department of Neonatology, Chengdu Women's and Children's Central Hospital, School of Medicine, University of Electronic Science and Technology of China, Chengdu, China

5 Department of Neonatology, Children's Hospital of Fudan University, National Children's Medical Center, Shanghai, China

6 Maternal-Infant Care Research Center (MiCARE) and Department of Pediatrics, Mount Sinai Hospital, Toronto, ON, Canada

7 Department of Pediatrics, Department of Obstetrics and Gynecology and Dalla Lana School of Public Health, University of Toronto, Toronto, ON, Canada 


\section{Introduction}

In December 2019, an outbreak of the coronavirus disease 2019 (COVID-19) caused by severe acute respiratory syndrome coronavirus 2 (SARS-CoV-2) was declared. Pregnant women and neonates are vulnerable to this virus because it is highly contagious [1-5]. To date, few neonatal COVID19 cases have been reported [1,6]. Thirty-three neonates born to mothers with COVID-19 in our present cohort were reported in a previous study, in which the majority of those exposed to SARS-CoV-2 were mildly symptomatic and had favorable outcomes during hospitalization [1]. However, epidemiological and clinical data remain limited, even as the large number of cases of COVID-19 continues to rise. In addition, cases of neurological complications have emerged in patients with coronaviruses [7-9]. A study by Helms and colleagues described neurological features in 58 adults admitted to hospital due to COVID-19, indicating that encephalopathy and ischemic strokes observed in brain magnetic resonance imaging (MRI) may have been due to critical illness or specific to SARS-CoV-2 infection [10]. Currently, rare neurological dysfunction has been noted in neonates with COVID-19. However, congenital infection or prenatal virus exposure, such as to Zika virus, appear to be associated with an increased risk of long-term neurologic sequelae [11]. Francis et al. suggested that prenatal infection or inflammation can be associated with poor motor development [12]. To our knowledge, there are no published studies reporting longitudinal neurologic assessments of neonates after COVID-19. Therefore, our primary objectives were (1) to identify whether abnormal brain MRI findings were evident in SARS-CoV-2-exposed neonates at a corrected gestational age of 44 weeks; (2) to identify whether the volumes of gray and white matter in the brain are changed in SARSCoV-2-exposed neonates at a corrected gestational age of 44 weeks; and (3) to investigate the physical growth development of SARS-CoV-2-exposed neonates at a corrected gestational age of 44 weeks. We present the following article in accordance with the Strengthening the Reporting of Observational Studies in Epidemiology reporting checklist.

\section{Methods}

\section{Study design and setting}

This was a multicenter observational study conducted at two children's hospitals in Hubei Province, mainland China (Wuhan Children's Hospital and Hubei Maternal and Child Health Hospital), from February 1, 2020 to May 15, 2020. Because SARS-CoV-2 is highly contagious to the general population, all suspected or confirmed patients are initially admitted to designated hospitals, including Wuhan Children's Hospital and Hubei Maternal and Child Health Hospital. In the present study, we targeted follow-up exams for all participants at a corrected gestational age of 44 weeks, to be carried out at local hospital clinics. The study was approved by the local institutional ethics committee at each participating hospital and informed written consent was obtained from the guardians of all participating neonates before the study began.

\section{Study population}

The neonates born to mothers with COVID-19 were recruited from Wuhan Children's Hospital and Hubei Maternal and Child Health Hospital from February 1, 2020 to May 15, 2020. We excluded neonates with major anomalies defined according to the US Centers for Disease Control and Prevention guidelines [13].

COVID-19 cases were confirmed based on the 7th edition of the New Coronavirus Pneumonia Prevention and Control Protocol for COVID-19 released by the National Health Commission of the People's Republic of China [15]. Discharge criteria were based on neonatal expert consensus [14] and the 7th edition of the New Coronavirus Pneumonia Prevention and Control Protocol for COVID-19 [15]. The early-onset infection is defined as the infection in the first week of life.

\section{Outcomes}

Our primary outcomes were: (1) MRI findings at 44 weeks' corrected gestational age, and grey matter and white matter brain volumes at 44 weeks' corrected gestational age and (2) physical growth parameters (weight, head circumference, and length) at 44 weeks' corrected gestational age. Our secondary outcomes were anti-SARS-CoV-2 immunoglobulin M (IgM) and IgG antibody statuses before delivery in mothers, and at birth, at the beginning of the 1st week of life, the beginning of 2 nd week of life, and the beginning of 4 th week of life in the neonates.

\section{Collection of clinical and laboratory data}

We used case report forms [16] from all recruited neonates to collect data on epidemiological history, demographics, clinical and radiological characteristics, laboratory parameters, and therapeutic regimens administered. Two researchers independently checked and recorded the data to ensure accuracy.

Brain MRI was carried out at the local participating institutions at a corrected gestational age of 44 weeks. Imaging was performed with a Siemens Magnetom Espree 1.5 T scanner (Siemens Medical System, Germany) using phased 
array surface coils. Infants were first fed, tightly swaddled, and had earplugs inserted; then they were scanned during natural sleep. If required, rectal chloral hydrate for sedation was administered with parental consent. Infants were monitored with an oxygen saturation probe. The images were analyzed by the pediatric neurologist and radiologists at the Children's Hospital of Fudan University, China. All T2-weighted imaging (T2WI) data were checked visually, and those with apparent artifacts, motion blurs, and outliers were excluded. For the 18 usable T2WI data (15 healthy newborns and 3 patients), we extracted the brain tissues from the whole head using FMRIB Software Library (FSL 6; http://www.fmrib. ox.ac.uk/fsl). Voxel-based morphometry analyses of the extracted T2WI data were performed using Statistical Parametric Mapping (SPM12; http://www.fil.ion.ucl.ac.uk/spm). We first normalized the extracted T2WI data to a standard template space of neonates, and segmented them into grey matter, white matter, and cerebrospinal fluid. We then smoothed the grey matter images using a Gaussian filter with a kernel of full width at half-maximum $=5 \mathrm{~mm}$.

Physical growth parameters were assessed in all neonates and included weight, length, and head circumferences. These were measured at birth and at corrected gestational age of 44 weeks using Fenton curves at local hospital clinics. However, owing to enforcement of strict, nationwide quarantine strategy during the study period, some neonates could not attend a local clinic and thus were evaluated by their parents. To facilitate this process, we created an instructional physical growth measurement video that taught parents how to take accurate measurements of their infant's weight, length, and head circumference. The parents took these measurements and gave them to us.

The SARS-CoV-2 infection status was tested by real-time reverse transcription polymerase chain reaction (RT-PCR) (novel coronavirus PCR fluorescence diagnostic kit; BGI, China) at the Chinese Center for Disease Control and Prevention. Procedures for the collection, transfer, and processing of the samples met the requirements of the World Health Organization. SARS-CoV-2 RT-PCR testing was performed for neonates at birth and after every 3 days until two consecutive results showed negative for SARS-CoV-2. RT-PCR testing was performed for mothers on admission and after every 3 days until two consecutive results showed negative for SARS-CoV-2.

Tests for IgM and IgG antibodies against SARS-CoV-2 (CMIA assays kit; Shenzhen InnoDx Biotech Co., Ltd., China) were conducted before delivery in the mothers and at birth, at the beginning of the 1st week of life, the beginning of 2 nd week of life, and the beginning of 4 th week of life in the neonates. The sensitivity and specificity reported by the manufacturer for IgM were $86.6 \%$ and $98.3 \%$, respectively; and for $\mathrm{IgG}$ were $96.3 \%$ and $97.4 \%$, respectively.

\section{Statistical analyses}

Descriptive statistics were used to establish clinical parameters in neonates exposed to SARS-CoV-2. For continuous variables, the means and standard deviations or medians and interquartile ranges (IQR), as appropriate, were reported; for categorical variables, percentages were reported. We performed a two-sample $t$ test on the smoothed grey matter images between control and case groups. The cutoff threshold value for statistical significance was set at $P<0.001$ (uncorrected) and a cluster size $>50$ voxels.

\section{Results}

\section{Demographic characteristics and baseline clinical features of SARS-CoV-2-exposed neonates}

The study population and numbers of enrolled neonates for which each type of outcome data are illustrated in Fig. 1. Table 1 reports the baseline information and clinical and radiologic features of the SARS-CoV-2-exposed neonates. During the study period, we identified 72 neonates eligible for study inclusion. Among these neonates (33 of whom were reported in a previous study [1]), $72(100 \%)$ were born to mothers with mild to moderate COVID-19 illness at third trimester, $53(74 \%)$ were delivered by cesarean section, 8 (11\%) were critically ill, and no deaths were reported. A median (IQR) age of admission was 20 (2-24) hours and a median (IQR) gestational age was 38 (37-39) weeks in these 72 neonates, and 31 (43\%) of them were male. Of 72 neonates exposed to SARS-CoV-2, 8 (11\%) were diagnosed with COVID-19. Of the eight neonates with COVID-19, 6 (75\%) had early-onset infection. There was no statistical difference in gestational age and weight compared between the two groups.

\section{Brain magnetic resonance imaging and growth outcomes}

\section{Neurodevelopment}

Figure 2 presents the results from reported brain MRI performed in eight SARS-CoV-2-exposed neonates including five neonates with COVID-19 and three without COVID19. Among five neonates with COVID-19, three (60\%) neonates presented brain MRI changes, including newborn 2 presented abnormal signals in white matter with delayed myelination, newborn 3 had brain hypoplasia with delayed myelination, and newborn 5 had abnormal signals in the bilateral periventricular indicating hypoxic changes. Compared with healthy newborns at the equivalent age, the volumes of grey matter (including cortical grey matter and deep 


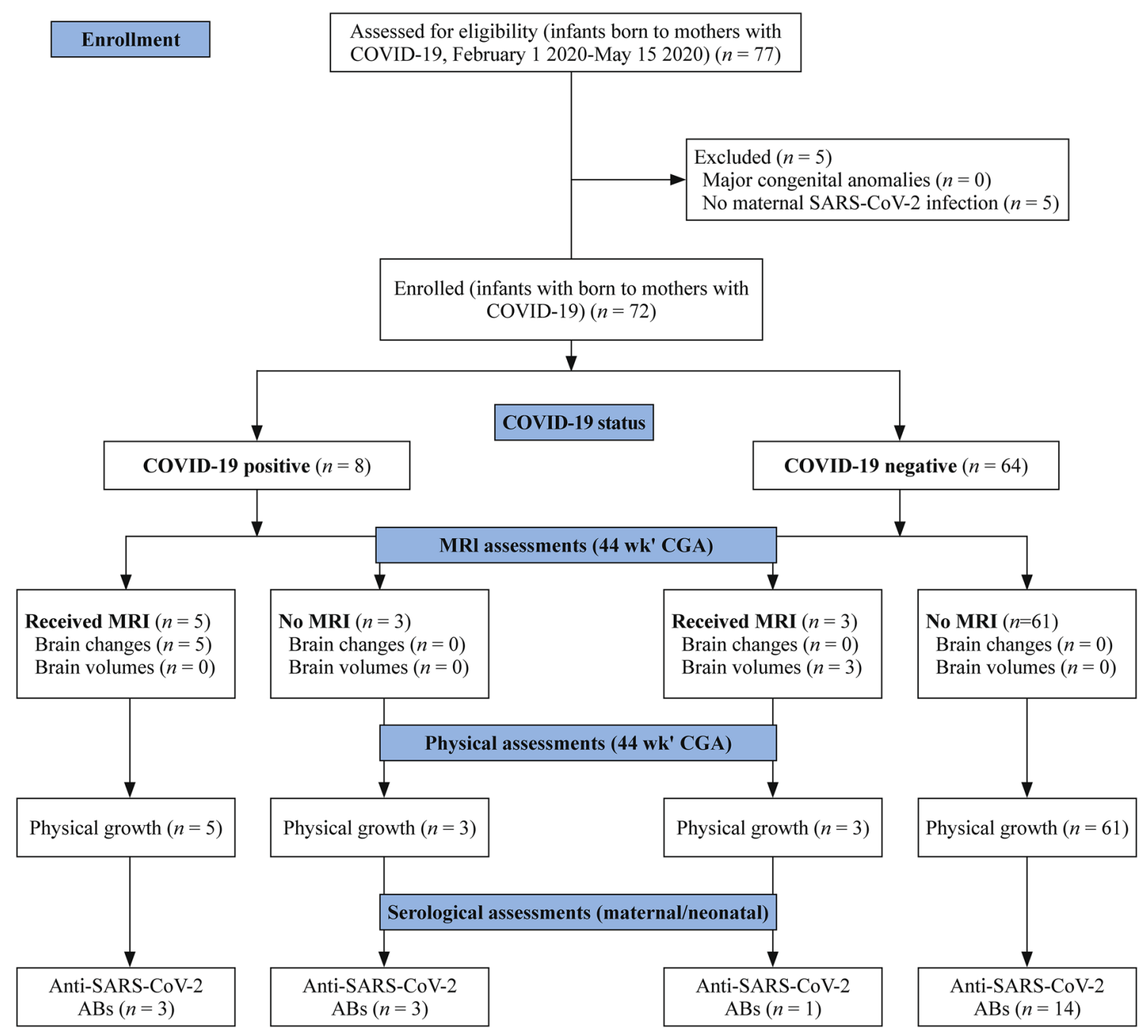

Fig. 1 Flow diagram of the study population. COVID-19 coronavirus disease 2019, SARS-CoV-2 severe acute respiratory syndrome coronavirus 2, MRI magnetic resonance imaging, $A B s$ antibodies

grey matter) and white matter and brain MRI findings in the three neonates without COVID-19 were not significantly different $(P>0.05)$.

\section{Physical growth}

Figure 3 illustrates the physical growth development of neonates exposed to SARS-CoV-2. The physical growth parameters in 72 SARS-CoV-2-exposed neonates at corrected gestational age of 44 weeks, including weight, length, and head circumference, were all above the 3 rd percentiles. No effects on physical growth were found in SARS-CoV2-exposed neonates.

\section{Dynamic changes in antibodies against SARS-CoV-2}

Of 21 SARS-CoV-2-exposed neonates assessed for antibodies against SARS-CoV-2, 6 (29\%) were positive for IgG antibodies at birth (Table 2). Supplementary Fig. 1a shows that no positive IgM antibodies were detected in five COVID-19-infected neonates who were SARS-CoV-2-positive by RT-PCR. Among them, newborn 5 with positive RT-PCR for SARS-CoV-2 was negative for IgG antibodies against SARS-CoV-2 at the 1 st week of life but was positive at the 2nd week of life. Newborn 7 with RT-PCR for SARS-CoV-2 with negative was positive for IgM antibodies against SARS-CoV-2 at birth but was negative at the 4th 
Table 1 Baseline information and clinical and lung radiologic features of neonates born to mothers with COVID-19

\begin{tabular}{|c|c|c|c|c|c|c|c|}
\hline \multirow[t]{2}{*}{ Characteristics or features } & \multirow{2}{*}{$\begin{array}{l}\text { Total } \\
(n=72)\end{array}$} & \multicolumn{3}{|c|}{ Neonates with COVID-19 } & \multicolumn{3}{|c|}{ Neonates without COVID-19 } \\
\hline & & $\begin{array}{l}\text { Total } \\
(n=8)\end{array}$ & $\begin{array}{l}\text { MRI } \\
(n=5)\end{array}$ & $\begin{array}{l}\text { No MRI } \\
(n=3)\end{array}$ & $\begin{array}{l}\text { Total } \\
(n=64)\end{array}$ & $\begin{array}{l}\text { MRI } \\
(n=3)\end{array}$ & $\begin{array}{l}\text { No MRI } \\
(n=61)\end{array}$ \\
\hline \multicolumn{8}{|l|}{ Maternal information } \\
\hline Lab-confirmed cases, $n(\%)$ & $52(72)$ & $8(100)$ & $5(100)$ & $3(100)$ & $44(69)$ & $3(100)$ & $41(67)$ \\
\hline Clinical diagnosed cases, $n(\%)$ & $20(28)$ & $0(0)$ & $0(0)$ & $0(0)$ & $20(31)$ & $0(0)$ & $20(33)$ \\
\hline Age $(y)$, mean (SD) & $29.88(3.44)$ & $29.29(3.25)$ & $29.60(3.78)$ & $28.30(1.52)$ & $29.95(3.48)$ & $33.00(2.00)$ & $29.70(3.48)$ \\
\hline Confirmed COVID-19 at third trimester, $n(\%)$ & $72(100)$ & $8(100)$ & $5(100)$ & $3(100)$ & $64(100)$ & $3(100)$ & $61(100)$ \\
\hline \multicolumn{8}{|l|}{ Disease severity status, $n(\%)$} \\
\hline Mild to moderate & $72(100)$ & $8(100)$ & $5(100)$ & $3(100)$ & $64(100)$ & $3(100)$ & $61(100)$ \\
\hline Severe or critical & $0(0)$ & $0(0)$ & $0(0)$ & $0(0)$ & $0(0)$ & $0(0)$ & $0(0)$ \\
\hline Delivery mode (CS) & $53(74)$ & $4(50)$ & $3(60)$ & $1(33)$ & $49(77)$ & $2(67)$ & $47(77)$ \\
\hline Meconium stained AF & $6(8)$ & $3(38)$ & $3(60)$ & $0(0)$ & $3(5)$ & $0(0)$ & $3(5)$ \\
\hline Premature rupture of membranes & $5(7)$ & $0(0)$ & $0(0)$ & $0(0)$ & $5(8)$ & $0(0)$ & $5(8)$ \\
\hline Chorioamnionitis & $0(0)$ & $0(0)$ & $0(0)$ & $0(0)$ & $0(0)$ & $0(0)$ & $0(0)$ \\
\hline Co-infected pathogens ${ }^{\mathrm{a}}$ & $7(10)$ & $0(0)$ & $0(0)$ & $0(0)$ & $7(11)$ & $0(0)$ & $7(11)$ \\
\hline \multicolumn{8}{|l|}{ Neonatal information } \\
\hline Age of admission to NICU (h), median (IQR) & $20(2-24)$ & $60(24-120)$ & $72(48-73)$ & $24(24-168)$ & $7.5(1.5-24)$ & $0(0-0)$ & $10(2-24)$ \\
\hline Gestational age (wk), median (IQR) & $38(37-39)$ & $\begin{array}{l}38.5(37.5- \\
39.5)\end{array}$ & $39(37-39)$ & $38(38-40)$ & $38(37-39)$ & $36(34-38)$ & $38(37-39)$ \\
\hline Male/female & $31 / 41$ & $3 / 5$ & $2 / 3$ & $1 / 2$ & $28 / 36$ & $1 / 2$ & $27 / 34$ \\
\hline Birth weight (kg), median (IQR) & $\begin{array}{l}3.31(3.00- \\
3.62)\end{array}$ & $\begin{array}{l}3.19(2.99- \\
3.36)\end{array}$ & $\begin{array}{l}3.35(3.03- \\
3.36)\end{array}$ & $\begin{array}{l}3.12(2.94- \\
3.25)\end{array}$ & $\begin{array}{l}3.05(2.65- \\
3.40)\end{array}$ & $\begin{array}{l}2.65(2.35- \\
2.95)\end{array}$ & $\begin{array}{l}3.08(2.74- \\
3.41)\end{array}$ \\
\hline Head circumference $(\mathrm{cm})$, mean (SD) & l & $33.63(3.32)$ & l & l & $33.93(1.91)$ & l & / \\
\hline Length $(\mathrm{cm})$, mean $(\mathrm{SD})$ & / & $48.9(3.75)$ & / & l & $48.51(3.15)$ & l & / \\
\hline Twins, $n(\%)$ & $4(6)$ & $1(13)$ & $1(20)$ & $0(0)$ & $3(5)$ & $0(0)$ & $3(5)$ \\
\hline SGA, $n(\%)$ & $2(3)$ & $0(0)$ & $0(0)$ & $0(0)$ & $2(3)$ & $0(0)$ & $2(3)$ \\
\hline Asphyxia, $n(\%)$ & $1(1)$ & $1(13)$ & $1(20)$ & $0(0)$ & $0(0)$ & $0(0)$ & $0(0)$ \\
\hline \multicolumn{8}{|l|}{ Clinical course } \\
\hline Asymptomatic cases, $n(\%)$ & $35(49)$ & $5(63)$ & $2(40)$ & $3(100)$ & $30(47)$ & $1(33)$ & $29(48)$ \\
\hline Symptomatic cases, $n(\%)$ & $37(51)$ & $3(38)$ & $3(60)$ & $0(0)$ & $34(53)$ & $2(67)$ & $32(52)$ \\
\hline Fever, $n(\%)$ & $13(18)$ & $3(38)$ & $2(40)$ & $0(0)$ & $10(16)$ & $0(0)$ & $10(16)$ \\
\hline Lethargy, $n(\%)$ & $6(8)$ & $1(13)$ & $1(20)$ & $0(0)$ & $5(8)$ & $0(0)$ & $5(8)$ \\
\hline Reported any respiratory symptoms, $n(\%)$ & $21(29)$ & $1(13)$ & $1(20)$ & $0(0)$ & $20(3)$ & $2(67)$ & $18(30)$ \\
\hline Reported any gastrointestinal symptoms, $n(\%)$ & $16(22)$ & $1(13)$ & $1(20)$ & $0(0)$ & $15(23)$ & $0(0)$ & $15(25)$ \\
\hline PCIS $<90, n(\%)$ & $8(11)$ & $1(13)$ & $1(20)$ & $0(0)$ & $7(11)$ & $0(0)$ & $7(11)$ \\
\hline Received oxygen support, $n(\%)$ & $5(7)$ & $0(0)$ & $0(0)$ & $0(0)$ & $5(8)$ & $0(0)$ & $5(8)$ \\
\hline Received ventilatory support, $n(\%)$ & $4(6)$ & $1(13)$ & $1(20)$ & $0(0)$ & $7(11)$ & $0(0)$ & $7(11)$ \\
\hline Received antibiotics, $n(\%)$ & $17(24)$ & $1(13)$ & $1(20)$ & $0(0)$ & $16(25)$ & $0(0)$ & $16(26)$ \\
\hline Received antiviral drugs, $n(\%)$ & $0(0)$ & $0(0)$ & $0(0)$ & $0(0)$ & $0(0)$ & $0(0)$ & $0(0)$ \\
\hline $\begin{array}{l}\text { Days to achieve discharge criteria (d), median } \\
(\text { IQR })^{\mathrm{b}}\end{array}$ & l & $\begin{array}{l}20.5(14.5- \\
27.5)\end{array}$ & $17(13-28)$ & $24(16-27)$ & $14(7.5-18)$ & $14(7-18)$ & $14(8-18)$ \\
\hline Early-onset COVID-19, $n(\%)$ & $6(8)$ & $6(75)$ & $4(80)$ & $2(67)$ & $0(0)$ & $0(0)$ & $0(0)$ \\
\hline Mortality, $n(\%)$ & $0(0)$ & $0(0)$ & $0(0)$ & $0(0)$ & $0(0)$ & $0(0)$ & $0(0)$ \\
\hline \multicolumn{8}{|l|}{ Lung radiologic features, $n(\%)$} \\
\hline Opacity or opacities, or densities & $14(19)$ & $2(25)$ & $2(40)$ & $0(0)$ & $12(19)$ & $0(0)$ & $12(20)$ \\
\hline Infiltrate & $18(25)$ & $6(75)$ & $3(60)$ & $3(100)$ & $12(19)$ & $2(67)$ & $10(16)$ \\
\hline Pneumonia & $20(30)$ & $6(75)$ & $5(100)$ & $1(33)$ & $14(22)$ & $2(67)$ & $12(20)$ \\
\hline Consolidation & $0(0)$ & $0(0)$ & $0(0)$ & $0(0)$ & $0(0)$ & $0(0)$ & $0(0)$ \\
\hline Ground-glass & $8(11)$ & $1(13)$ & $1(20)$ & $0(0)$ & $7(11)$ & $0(0)$ & $7(11)$ \\
\hline Pneumothorax & $1(1)$ & $0(0)$ & $0(0)$ & $0(0)$ & $1(2)$ & $1(33)$ & $0(0)$ \\
\hline
\end{tabular}


Table 1 (continued)

\begin{tabular}{|c|c|c|c|c|c|c|c|}
\hline \multirow[t]{2}{*}{ Characteristics or features } & \multirow{2}{*}{$\begin{array}{l}\text { Total } \\
(n=72)\end{array}$} & \multicolumn{3}{|c|}{ Neonates with COVID-19 } & \multicolumn{3}{|c|}{ Neonates without COVID-19 } \\
\hline & & $\begin{array}{l}\text { Total } \\
(n=8)\end{array}$ & $\begin{array}{l}\text { MRI } \\
(n=5)\end{array}$ & $\begin{array}{l}\text { No MRI } \\
(n=3)\end{array}$ & $\begin{array}{l}\text { Total } \\
(n=64)\end{array}$ & $\begin{array}{l}\text { MRI } \\
(n=3)\end{array}$ & $\begin{array}{l}\text { No MRI } \\
(n=61)\end{array}$ \\
\hline Effusion & $3(4)$ & $0(0)$ & $0(0)$ & $0(0)$ & $3(5)$ & $0(0)$ & $3(5)$ \\
\hline Transient tachypnea of newborn & $0(0)$ & $0(0)$ & $0(0)$ & $0(0)$ & $0(0)$ & $0(0)$ & $0(0)$ \\
\hline Other lab-confirmed pathogens, $n(\%)^{\mathrm{c}}$ & $2(3)$ & $1(13)$ & $1(20)$ & $0(0)$ & $1(2)$ & $0(0)$ & $1(2)$ \\
\hline \multicolumn{8}{|l|}{ Initial laboratory results, $n(\%)$} \\
\hline White blood cells $<5$ or $>30\left(\times 10^{9} / \mathrm{L}\right)$ & $5(7)$ & $1(13)$ & $1(20)$ & $0(0)$ & $4(6)$ & $0(0)$ & $4(7)$ \\
\hline Lymphocytes $<2\left(\times 10^{9} / \mathrm{L}\right)$ & $10(14)$ & $1(13)$ & $1(20)$ & $0(0)$ & $9(14)$ & $0(0)$ & $9(15)$ \\
\hline Platelets $<150\left(\times 10^{12} / \mathrm{L}\right)$ & $2(3)$ & $1(13)$ & $1(20)$ & $0(0)$ & $1(2)$ & $0(0)$ & $1(2)$ \\
\hline Elevated C-reactive protein $(0-3 \mathrm{mg} / \mathrm{dL})$ & $7(10)$ & $1(13)$ & $1(20)$ & $0(0)$ & $6(9)$ & $0(0)$ & $6(10)$ \\
\hline Elevated IL-6 (0-20.9 mg/dL) & $14(19)$ & $2(25)$ & $1(20)$ & $1(33)$ & $12(19)$ & $0(0)$ & $12(20)$ \\
\hline Elevated PCT $(<0.1 \mathrm{mg} / \mathrm{dL})$ & $10(14)$ & $3(38)$ & $2(40)$ & $1(33)$ & $7(11)$ & $0(0)$ & $7(11)$ \\
\hline
\end{tabular}

COVID-19 coronavirus disease 2019, SD standard deviation, $C S$ cesarean section, $A F$ amniotic fluid, NICU neonatal intensive care unit, IQR interquartile range, $S G A$ small for gestational age, $P C I S$ pediatric critical illness score, $I L$ interleukin, $P C T$ procalcitonin. ${ }^{a}$ Maternal co-infected pathogens: IgG antibodies for Mycoplasma pneumoniae were detected in seven mothers with COVID-19. TORCH or GBS screens were all negative; ${ }^{b} \mathrm{Six}$ asymptomatic neonates born to mothers with COVID-19 were not admitted to the neonatology department. Of 72 neonates, five were discharged at the parents' request (they did not achieve the discharge criteria); ${ }^{c}$ Neonatal co-infected pathogens: one neonate with COVID19 had an Enterobacter agglomerates-positive blood culture and one neonate without COVID-19 had an Escherichia coli-positive blood culture

week of life (Supplementary Fig. 1b). Only two neonates (newborn 6 and newborn 7) remained positive for IgG antibodies against SARS-CoV-2 at 4 weeks after birth (Supplementary Fig. 1a).

\section{Discussion}

To our knowledge, this study is the first to fully describe the clinical course of neonates born to mothers with COVID19 including the clinical manifestations, short-term physical growth development and MRI findings, and dynamic change of antibodies against SARS-CoV-2. Consistent with the previous study [1], we found that most neonates born to mothers with COVID-19 were asymptomatic for COVID-19 or mild illness, and eight (11\%) neonates born to mothers with COVID-19 had positive RT-PCR for SARS-CoV-2 or IgM against SARS-CoV-2, but most of them were asymptomatic.

Moreover, this study revealed that neonates born to mothers with COVID-19 could have abnormal MRI findings at a corrected gestational age of 44 weeks while also having normal grey and white matter brain volume and favorable physical growth. We identified three neonates with COVID19 presenting brain MRI abnormalities. Except for positive SARS-CoV-2 PCR and persistent IgG antibodies against SARS-CoV-2, no other risk factors affecting neurological development were found in the two full-term neonates (newborn 2 and newborn 5). Newborn 3, who was reported in the previous study [1], was born at a gestational age of $31+2$ weeks. There were no acute neurological symptoms in these three neonates, but abnormal brain MRI findings were reported. Other studies suggested that brain MRI parenchymal signal abnormalities were associated with COVID-19 in adults [17]. Therefore, our findings highlight that neurological systems of neonates with COVID-19 could be involved and should be monitored closely. On the other hand, studies have shown that white matter and cerebellar volumes at term gestational age were associated with cognitive, language, and motor outcomes [18]. Therefore, we further investigated the grey and white matter volumes of three neonates born to mothers with COVID-19; however, compared to the healthy newborns, no difference in volume was noted. Owing to limitations related to follow-up time and the numbers of individuals available for follow-up and for MRI testing, it is still unknown whether the SARS-CoV-2 virus affects the long-term neural development in neonates born to mothers with COVID-19.

We observed low rates of positive transplacental $\mathrm{IgG}$ antibodies in neonates born to mothers with COVID-19, possibly because the mothers were infected during the 3rd trimester. Although the serum $\operatorname{IgG}$ antibodies for SARS$\mathrm{CoV}-2$ in these newborns could last for at least 3 weeks, it remains uncertain whether the positive $\mathrm{IgG}$ antibodies could be protective and whether the duration of transplacental IgG 


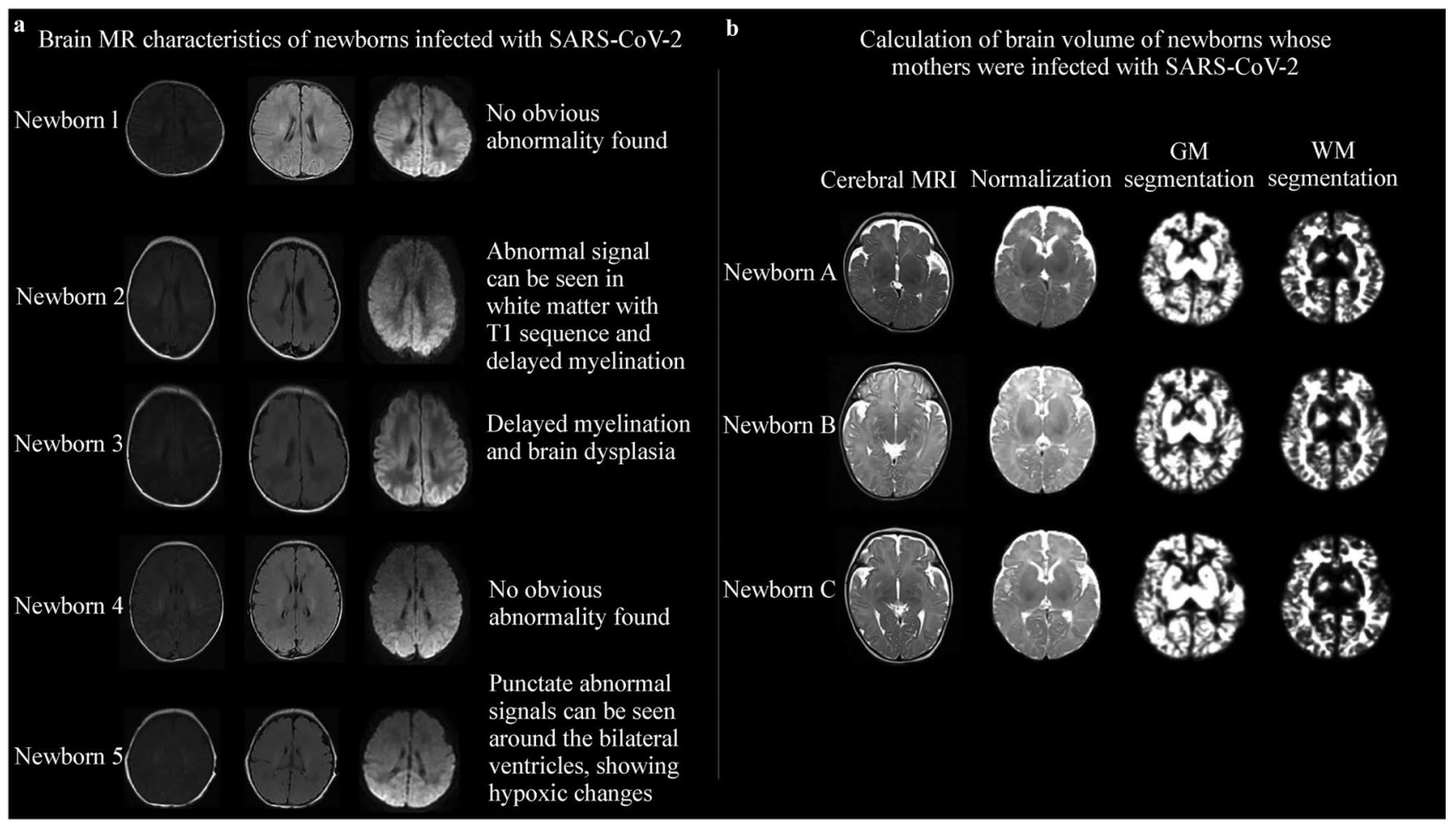

Fig. 2 Neurological development of neonates born to mothers with COVID-19. a Newborn 1: GA 40+4 weeks, BW 3360 g. She had positive RT-PCR for SARS-CoV-2. She was admitted to NICU at day of birth and was asymptomatic. She was discharged home at 17 days after birth. Newborn 2: GA 37+2 weeks, BW 2940 g. No prenatal hypoxic history was reported. He had only positive RT-PCR for SARS-CoV-2. He was admitted to NICU at 18 days after birth. He presented fever $\left(37.8{ }^{\circ} \mathrm{C}\right)$ and was discharged home at 28 days of life. Newborn 3: GA $31+2$ weeks BW $1580 \mathrm{~g}$. She was positive RT-PCR for SARS-CoV-2 at birth but negative IgM and IgG against SARS-CoV-2 at 4 weeks after birth [1]. Newborn 4: GA 39 weeks, BW 3350 g. He had positive RT-PCR for SARS-CoV-2 at 7 days after birth and positive IgG against SARS-CoV-2 at 4 weeks after birth. He was admitted to NICU

antibody expression depends on the maternal serum level of IgG antibodies. Newborn 5 with positive SARS-CoV-2 PCR at birth was negative for IgG antibodies initially but was positive at 2 weeks after birth, indicating the early-onset neonatal COVID-19 infection but vertical transmission was still suspicious. In our study, although the RT-PCR test for SARS-CoV-2 was negative in newborn 7 , she was diagnosed as positive for both IgM and IgG antibodies against SARS-CoV-2 at birth based on the current protocol [15]. At present, serum testing may play a complementary role for diagnosing COVID-19. at 7 days after birth. He presented fever $\left(37.7^{\circ} \mathrm{C}\right)$ and was discharged home at 20 days after birth. Newborn 5: GA 39+1 week, BW 3570 g. No prenatal hypoxic history was reported. She had positive RT-PCR for SARS-CoV-2 but negative IgM and IgG against SARS-CoV-2 at 1 week after birth and positive IgG against SARS-CoV-2 at 2 weeks after birth. She was admitted to NICU at 3 days after birth and was asymptomatic. She was discharged home at 12 days after birth; b Newborn A, B, and C without COVID-19 were only born to mothers with COVID-19 and they were term infants and asymptomatic. COVID-19 coronavirus disease 2019, SARS-CoV-2 severe acute respiratory syndrome coronavirus 2, $G A$ gestational age, $B W$ birth weight, $R T-P C R$ real-time reverse transcription polymerase chain reaction, $N I C U$ neonatal intensive care unit, Ig immunoglobulin, MRI magnetic resonance imaging

Several limitations in our study might create bias. First, this was a short-term follow-up study, whereas a long-term followup would provide more information, such as whether neonates with abnormal MRI findings would present with cognitive, language, and motor disorders in later life. Second, the number of neonates born to mothers with COVID-19 who underwent brain MRI assessment was small and generalization from this subset may be limited. We did not have more cranial MR data because we did not obtain more neonatal infections. Our results should be interpreted with caution because they reflect individual neurologic assessments and imaging performed 

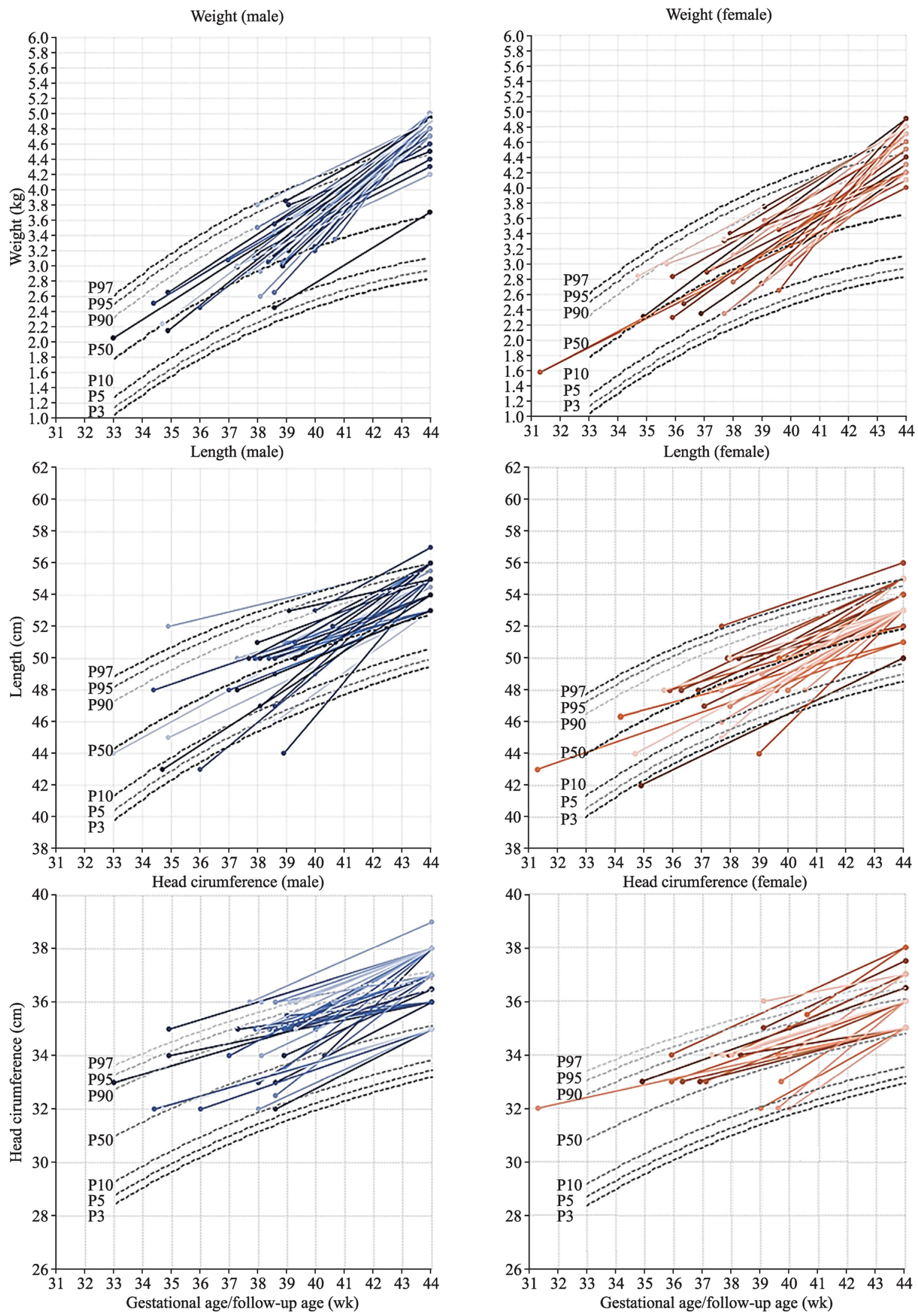

Fig. 3 Short-term physical growth curves of the neonates born to mothers with coronavirus disease 2019. $P 3$ 3rd percentiles, $P 55$ th percentile, $P 10$ 10th percentile, $P 50$ 50th percentile, $P 9090$ th percentile, $P 9595$ th percentile, $P 9797$ th percentile 
Table 2 Primary and secondary outcomes by neonate COVID-19 and MRI assessment status

\begin{tabular}{|c|c|c|c|c|}
\hline \multirow[t]{2}{*}{ Outcomes } & \multicolumn{2}{|c|}{ Neonates with COVID-19 $(n=8)$} & \multicolumn{2}{|c|}{ Neonates without COVID-19 $(n=64)$} \\
\hline & Received MRI $(n=5)$ & No MRI $(n=3)$ & Received MRI $(n=3)$ & No MRI $(n=61)$ \\
\hline White matter changes, $n(\%)$ & $3(60)$ & NA & NA & NA \\
\hline Physical growth at 44 wk CGA, $n(\%)$ & $5(100)$ & $3(100)$ & $3(100)$ & $61(100)$ \\
\hline Weight (kg), median (IQR) & $4.6(4.4-4.7)$ & $4.3(4.3-4.5)$ & $4.3(4.2-5.5)$ & $4.5(4.3-5.0)$ \\
\hline Length $(\mathrm{cm})$, median $(\mathrm{IQR})$ & $53(53-55)$ & $53(53-53)$ & $54(52-56)$ & $54(53-55)$ \\
\hline Head circumference $(\mathrm{cm})$, median (IQR) & $36(36-36)$ & $35(35-36)$ & $35(35-38)$ & $36(36-37)$ \\
\hline $\begin{array}{l}\text { Serological assessments }{ }^{\mathrm{a}} \text { (anti-SARS-CoV-2 } \\
\text { antibodies), } n(\%)\end{array}$ & $3(60)$ & $3(100)$ & $1(33)$ & $14(23)$ \\
\hline \multicolumn{5}{|l|}{ Maternal, $n(\%)$} \\
\hline IgG-positive before birth & $3(100)$ & $3(100)$ & $1(100)$ & $14(100)$ \\
\hline IgM-positive before birth & $0(0)$ & $2(67)$ & $1(100)$ & $4(31)$ \\
\hline \multicolumn{5}{|l|}{ Neonatal } \\
\hline \multicolumn{5}{|l|}{ IgG-positive, $n(\%)$} \\
\hline At birth & NA & $2(67)$ & NA & $3(21)$ \\
\hline 1 wk after birth & NA & $2(67)$ & NA & NA \\
\hline 2 wk after birth & $1(33)$ & $2(67)$ & $1(100)$ & NA \\
\hline 4 wk after birth & $1(33)$ & $2(67)$ & NA & NA \\
\hline \multicolumn{5}{|l|}{ IgM-positive, $n(\%)$} \\
\hline At birth & $0(0)$ & $1(33)$ & $0(0)$ & $0(0)$ \\
\hline 1 wk after birth & $0(0)$ & $1(33)$ & NA & NA \\
\hline 2 wk after birth & $0(0)$ & $1(33)$ & NA & NA \\
\hline 4 wk after birth & $1(33)$ & $0(0)$ & NA & NA \\
\hline
\end{tabular}

MRI assessments at 44 weeks CGA. COVID-19 coronavirus disease 2019, SARS-CoV-2 severe acute respiratory syndrome coronavirus 2 , NA not

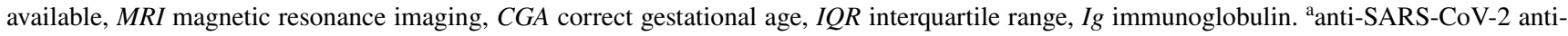
body expression was evaluated in 21 of the 72 maternal-neonatal pairs

in early infancy. More cases from other countries or regions would aid the interpretation of the neurologic findings in brain MRI of neonates born to mothers with COVID-19.

To our knowledge, this is the first study to describe the brain MRI and physical growth of neonates born to mothers with COVID-19. Our results suggested that there was some evidence of abnormal brain MRI findings in neonates with COVID-19, but there was no evidence of abnormal growth development in neonates born to mothers with COVID-19. At present, we cannot conclude that the abnormal brain MRI findings were caused by SARSCoV-2; therefore, the neonates born to mothers with COVID-19 could be followed-up as routine health care schedule but the routine neurodevelopmental surveillance of the neonates with COVID-19 should be evaluated by further study.

Supplementary Information The online version contains supplementary material available at https://doi.org/10.1007/s12519-021-00426-z.

Acknowledgements The authors thank all the study participants and their families. We thank Heather McDonald Kinkaid, PhD, from the
Maternal-infant Care Research Center (MiCare) at Mount Sinai Hospital in Toronto, Ontario, for editorial assistance in the preparation of this manuscript. MiCare is supported by a Canadian Institute of Health Research Team Grant (CTP 87518), the Ontario Ministry of Health and Long-Term Care, and the participating hospitals. We thank Prof. WeiLi Yan, Yin Wang, PhD, and Yi Zhang, MPh, from the Department of Clinical Epidemiology at Children's Hospital of Fudan University in Shanghai, China, for designing the maternal and neonatal case report forms.

Author contributions ZLK, ZHP and XTT contributed equally to this paper and should be considered as co-first author. ZWH, LSK, XSW, ZLK, XTT, YK, and ZHP conceptualized the study and analyzed the data. PSC, YWH, SJB, WLS, and XFF co-conceptualized the study and interpreted the data. All authors drafting the article and revising it critically for important intellectual content. All authors revised and approved the final manuscript.

Funding Three neonates who underwent brain MRI were supported by the Fundamental Research Funds for the Central Universities (No. 2020kfyXGYJ006) and Wuhan Emergency Research Project of COVID-19 (No. EX20D24). The funding bodies played no role in the design or conduct of the study; the collection, management, analysis, or interpretation of the data; the preparation, review, or approval of the manuscript; or the decision to submit the manuscript for publication. 


\section{Compliance with ethical standards}

Ethical approval The study was approved by the local institutional ethics committee at each participating hospital and informed written consent was obtained from the guardians of all participating neonates before the study began.

Conflict of interest No financial or nonfinancial benefits have been received or will be received from any party related directly or indirectly to the subject of this article. The authors have no conflict of interest to declare.

\section{References}

1. Zeng LK, Xia SW, Yuan WH, Yan K, Xiao FF, Shao JB, et al. Neonatal early-onset infection with SARS-CoV-2 in 33 neonates born to mothers with COVID-19 in Wuhan, China. JAMA Pediatr. 2020;174:722-5.

2. Chen HJ, Guo JJ, Wang C, Luo F, Yu XC, Zhang W, et al. Clinical characteristics and intrauterine vertical transmission potential of COVID-19 infection in nine pregnant women: a retrospective review of medical records. Lancet. 2020;395:809-15.

3. Zhu HP, Wang L, Fang CZ, Peng SC, Zhang LH, Chang GP, et al. Clinical analysis of 10 neonates born to mothers with 2019-nCoV pneumonia. Transl Pediatr. 2020;9:51-60.

4. Sutton D, Fuchs K, D'Alton M, Goffman D. Universal screening for SARS-CoV-2 in women admitted for delivery. N Engl J Med. 2020;382:2163-4.

5. Xiao FF, Chen B, Xiao TT, Lee SK, Yan K, Hu LY. Children with SARS-CoV-2 infection during an epidemic in China (outside of Hubei province). Ann Transl Med. 2020;8:849.

6. Munoz AC, Nawaratne U, McMann D, Ellsworth M, Meliones J, Boukas K. Late-onset neonatal sepsis in a patient with COVID-19. N Engl J Med. 2020;382:e49.

7. Netland J, Meyerholz DK, Moore S, Cassell M, Perlman S. Severe acute respiratory syndrome coronavirus infection causes neuronal death in the absence of encephalitis in mice transgenic for human ACE2. J Virol. 2008;82:7264-75.
8. Huang CL, Wang YM, Li XW, Ren LL, Zhao JP, Hu Y, et al. Clinical features of patients infected with 2019 novel coronavirus in Wuhan, China. Lancet. 2020;395:497-506.

9. Kim JE, Heo JH, Kim HO, Song SH, Park SS, Park TH, et al. Neurological complications during treatment of middle east respiratory syndrome. J Clin Neurol. 2017;13:227-33.

10. Helms J, Kremer S, Merdji H, Clere-Jehl R, Schenck M, Kummerlen C, et al. Neurologic features in severe SARS-CoV-2 infection. N Engl J Med. 2020;382:2268-70.

11. Adachi K, Nielsen-Saines K. Zika clinical updates: implications for pediatrics. Curr Opin Pediatr. 2018;30:105-16.

12. Francis F, Bhat V, Mondal N, Adhisivam B, Jacob S, Dorairajan $\mathrm{G}$, et al. Fetal inflammatory response syndrome (FIRS) and outcome of preterm neonates-a prospective analytical study. J Matern Fetal Neonatal Med. 2019;32:488-92.

13. Rasmussen SA, Olney RS, Holmes LB, Lin AE, Keppler-Noreuil $\mathrm{KM}$, Moore CA, et al. Guidelines for case classification for the National Birth Defects Prevention Study. Birth Defects Res A Clin Mol Teratol. 2003;67:193-201.

14. Wang LS, Shi Y, Xiao TT, Fu JH, Feng X, Mu DZ, et al. Chinese expert consensus on the perinatal and neonatal management for the prevention and control of the 2019 novel coronavirus infection (first edition). Ann Transl Med. 2020;8:47.

15. National Health Commission of the People's Republic of China. New coronavirus pneumonia prevention and control program, 7th ed. 2020. http://www.nhc.gov.cn/yzygj/s7653p/202003/46c92 94a7dfe4cef80dc7f5912eb1989.shtml. Accessed 4 Mar 2020.

16. Xiao TT, Xia SW, Zeng LK, Lin G, Wei QF, Zhou W, et al. A multicentre observational study on neonates exposed to SARSCoV-2 in China: the Neo-SARS-CoV-2 Study protocol. BMJ Open. 2020;10:e038004.

17. Kremer S, Lersy F, de Seze J, Ferre JC, Maamar A, Carsin-Nicol $\mathrm{B}$, et al. Brain MRI findings in severe COVID-19: a retrospective observational study. Radiology. 2020;297:E242-51.

18. Cheong JL, Thompson DK, Spittle AJ, Potter CR, Walsh JM, Burnett AC, et al. Brain volumes at term-equivalent age are associated with 2-year neurodevelopment in moderate and late preterm children. J Pediatr. 2016;174:91-7.e1.

Publisher's Note Springer Nature remains neutral with regard to jurisdictional claims in published maps and institutional affiliations. 